\title{
Análise Crítica dos Pacientes Cirúrgicos Internados na Unidade de Terapia Intensiva*
}

\section{Critical Analysis of Hospital Surgical Patients in Intensive Care Unit}

\author{
Cristina Sayuri Nakano', Nadim Farid Safatle², Marcelo Moock ${ }^{3}$
}

\section{RESUMO}

JUSTIFICATIVA E OBJETIVOS: A performance de cada unidade de terapia intensiva (UTI) precisa ser observada no contexto dos cuidados médicos, assim como pela instituição que ela faz parte. Existem vários tipos sistemas de índices prognósticos em terapia intensiva. O APACHE II foi introduzido por Knaus e col. em 1985, sendo um sistema amplamente usado para avaliar a gravidade dos pacientes em tratamento intensivo. O objetivo deste estudo foi avaliar o índice prognóstico (APACHE II) dos pacientes submetidos a cirurgia do aparelho digestivo de urgência ou eletiva encaminhadas para a UTI.

MÉTODO: Foram coletados os seguintes dados: idade, sexo, tempo de internação, indicação de UTI, tipo de cirurgia (eletiva ou de urgência), complicações, índice de massa corpórea (IMC), APACHEll e risco de óbito.

RESULTADOS: Foram analisados dados de 38 pacientes, no período de abril de 2005 a abril de 2006, dentre os quais 18 faleceram e 20 sobreviveram. A idade no grupo dos não sobreviventes variou de 44 a 92 com mé-

\footnotetext{
1. Residente de Cirurgia Geral

2. Professor Titular e Chefe da Disciplina de Clínica Cirúrgica da Faculdade de Medicina da Universidade de Santo Amaro

3. Professor Assistente e Chefe da Disciplina de Medicina Intensiva da Faculdade de Medicina da Universidade de Santo Amaro

*Recebido da Unidade de Terapia Intensiva do Hospital Liberato John Alphonse Di Dio da Faculdade de Medicina Santo Amaro, São Paulo, SP
}

Apresentado em 04 de maio de 2007

Aceito para publicação em 20 de julho de 2007

Endereço para correspondência:

Dra. Cristina Sayuri Nakano

R. Dom Sebastião do Rego, 426

04129-000 São Paulo, SP

Fone: (11) 5062-1069

E-mail: saynakano@hotmail.com

(C)Associação de Medicina Intensiva Brasileira, 2007 dia de 66,61 anos; enquanto no grupo dos sobreviventes a idade variou de 28 a 78 com média de 59,15 anos. Já em relação ao IMC no grupo dos não sobreviventes variou de 22 a 29 com média de 26; enquanto nos sobreviventes a média foi de 25,6. Não houve diferença significativa entre idade e IMC no grupo dos sobreviventes em relação ao grupo dos não sobreviventes. $O$ tempo de internação variou de 2 a 52 dias no grupo dos não sobreviventes com média de 11,3 dias. Enquanto no grupo dos sobreviventes a variação foi de 1 a 30 dias com média de 4,9. Em relação ao APACHE II no grupo dos não sobreviventes variou de 5 a 32 com média de 19,14. Ao passo que no grupo dos sobreviventes o APACHE variou de 1 a $18 \mathrm{com}$ média de 8,6. O tempo de internação e o APACHE II apresentaram diferenças significativas em ambos os grupos, sendo maior no grupo dos não sobreviventes. O risco de óbito no grupo dos não sobreviventes variou de 3,1 a 84,9 com média de 38,8 ; enquanto no grupo dos sobreviventes a média foi de 7,5 Neste estudo foi calculada a Standardized Mortality Rate (SMR razão da mortalidade observada pela predita), que teve como resultado 1,22.

CONCLUSÕES: Os pacientes não sobreviventes apresentaram APACHE II significativamente maior que os sobreviventes; maior tempo de internação dos pacientes não sobreviventes em relação aos sobreviventes; a SMR encontrou-se dentro da observada na literatura; não houve diferença estatística em relação ao IMC nos dois grupos.

Unitermos: APACHE II, pacientes cirúrgicos

\section{SUMMARY}

BACKGROUND AND OBJECTIVES: Each intensive care units result has to be observed in the context of medical care, as well as the institution witch it belongs. There are many types of prognostic index in intensive care. The APACHE II was introduced by Knaus et al. in 1985, being a widely used system to evaluate the illness severity in intensive care patients. This objective 
was evaluated the prognostic index (APACHE II) in patients submitted to elective or emergency gastrointestinal surgery admitted to the ICU.

METHODS: Medical school intensive care unit. It was collected the following data: age, sex, length of stay, intensive care indication, type of surgery (elective or emergency), body mass index (BMI) APACHE II and predicted mortality.

RESULTS: A total of 38 patients data were collected during the period of April 2005 to April 2006. Eighteen patients died and twenty survived. The age of the nonsurvivors varied from 44 to 92 (mean age 66.6); while the age of the survivors varied from 28 to 78 (mean age 59.1). The BMI of the non-survivors varied from 22 to 29 (mean body mass index 26) while in the other group the mean body mass index was 25.6. No significant difference was noted in the age and body mass index of both groups. The length of stay varied from 2 to 52 days in the non-survivors group (mean 11.3 days), while in the survivors group varied from 1 to 30 days (mean 4.9). The APACHE II varied from 5 to 32 in the non-survivors group (mean 19.14). While in the survivors group varied from 1 to 18 (mean 8.6). The length of stay and APACHE II demonstrated a significant difference in both groups, being higher in the non-survivors group. The non-survivors predicted mortality varied from 3.1 to 84.9 (mean 38.8); while the survivors mean was 7.5 . The Standardized Mortality Rate in this study was 1.22.

CONCLUSIONS: Non-survivors patients APACHE ॥ was higher than the survivors; the SMR was similar to others studies; the length of stay was longer in the nonsurvivors group; no statistic difference was noted in the body mass index.

Key Words: APACHE II, surgical patients

\section{INTRODUÇÃO}

Desde 1950 a terapia intensiva é considerada uma forma reconhecida de tratamento apresentando mudanças consideráveis até hoje. A introdução de novos tratamentos, o aumento da expectativa de vida e o crescimento das doenças crônicas que em determinado momento necessitam de cuidados intensivos, são alguns dos fatores que determinaram as rápidas mudanças dessa especialidade 1 .

Em 1971, foi inaugurada a primeira unidade de terapia intensiva (UTI) do Brasil, no Hospital Sírio-Libanês, sendo considerada o berço de unidades congêneres em outras instituições ${ }^{2}$.

No final da década de 1990, aproximadamente 95\% dos hospitais americanos apresentavam pelo menos uma UTI, existindo uma tendência para seu aumento e multiplicação³.

A performance de cada UTI precisa ser observada no contexto dos cuidados médicos, assim como pela instituição que faz parte. Mecanismos de avaliação do tratamento intensivo foram desenvolvidos, sendo reconhecidos mundialmente pela literatura científica. Esses métodos são chamados de índices de prognóstico. Eles podem ser usados independentemente ou associados com outras classificações. Estimativas do grau de gravidade e previsão da evolução dos pacientes críticos têm aplicações relevantes que permitem a avaliação da eficácia, análise dos custos e benefício do tratamento, permitindo tomar decisões de quando iniciar, manter ou interromper os cuidados intensivos ${ }^{1}$. Existem vários tipos sistemas de índices prognósticos em terapia intensiva. O primeiro deles é datado de 1974 conhecido como Therapeutic Intervention Scoring System (TISS). Desde então foram criados vários outros índices prognósticos sendo os principais o Acute Physiological and Chronic Health Evaluation (APACHE), o Simplified Acute Physiology Score (SAPS), o Mortality Probability Mode (MPM), o Multiple Organ Dysfunction Score (MODS), o Logistic Organ Dysfunction System (LODS) e finalmente o Sequential Organ Failure Assessment Score (SOFA) ${ }^{2}$.

O APACHE II foi introduzido por Knaus e col. em 1985, sendo um sistema amplamente usado para avaliar a gravidade dos pacientes em terapia intensiva ${ }^{4}$.

O objetivo deste estudo foi realizar análise crítica dos pacientes submetidos a cirurgia do aparelho digestivo de urgência ou eletiva encaminhados para a UTI.

\section{MÉTODO}

Foi realizado um estudo retrospectivo com dados de prontuários de pacientes submetidos à cirurgia do aparelho digestivo de urgência ou eletiva no período de abril de 2005 a abril de 2006, encaminhados à UTI do Hospital Liberato John Alphonse Di Dio da Faculdade de Medicina Santo Amaro.

Foram coletados os seguintes dados: idade, sexo, tempo de internação, indicação de UTI, tipo de cirurgia (eletiva ou de urgência), complicações, índice de massa corpórea (IMC), APACHE II e risco de óbito.

O sistema preditivo de mortalidade intra-hospitalar para pacientes graves, desenvolvido por Knaus e col, o Acute Phisiology and Cronic Health Evaluation II (APACHE II), foi utilizado como por eles descrito nos 
pacientes cirúrgicos internados na UTI deste hospital ${ }^{4}$. O APACHE II usa 11 medidas fisiológicas: pressão sanguínea, freqüência cardíaca ( $F C)$, freqüência respiratória $(\mathrm{FR})$, temperatura corporal, sódio sérico, potássio, creatinina, ph arterial, gradiente de oxigênio alveolararterial, hematócrito, contagem de leucócitos ${ }^{5}$. Além desses dados o índice de coma Glasgow também é avaliado.

A pontuação máxima é de 71 pontos, assim obtidos: idade, máxima de 6 pontos; o parâmetro fisiológico agudo: máximo 60 pontos e avaliação do estado prévio de saúde e local procedência imediata, máxima de 5 pontos (pacientes não-cirúrgicos ou submetidos a cirurgia de urgência com disfunção orgânica prévia receberam 5 pontos, pacientes submetidos a cirurgia eletiva 2 pontos) ${ }^{6}$.

A equação final para o cálculo do risco de óbito é expressa: $\operatorname{Ln}(R / 1-R)=-3,517+$ (APACHE II escore $x$ $0,146)+(0,603$ somente se cirurgia de emergência $)+$ (diagnóstico específico), onde $\mathrm{Ln}=$ logaritmo natural, $\mathrm{R}=$ risco de óbito. O risco de óbito expressa o valor preditivo de mortalidade em termos percentuais, de modo que o risco de óbito de 50 reflete o prognóstico de mortalidade de $50 \%{ }^{6}$.

Os pacientes foram divididos em 2 grupos: sobreviventes e não sobreviventes. Para a análise dos resultados foi aplicado o teste de Mann-Whitney (Siegel) comparando todas as variáveis estudadas no grupo não sobreviventes e no grupo sobrevivente. Fixou-se em 0,05 ou $5 \%$ o nível de rejeição da hipótese de nulidade ${ }^{7}$.

\section{RESULTADOS}

Foram analisados dados de 38 pacientes. Dezoito faleceram (mortalidade de 47\%) e 20 sobreviveram; dos não sobreviventes houve predomínio do sexo masculino (10), em relação ao sexo feminino (8). No grupo dos sobreviventes (20), 14 eram do sexo masculino e 6 do sexo feminino.

O órgão mais acometido foi o cólon com 15 pacientes. Em seguida a vesícula biliar com cinco pacientes, o fígado com quatro pacientes, o apêndice cecal, esôfago, estômago e intestino delgado foram acometidos na mesma freqüência com três pacientes. Por último o pâncreas foi acometido em dois pacientes.

A idade no grupo dos não sobreviventes variou de 44 a 92 com média de 66,61 anos; enquanto no grupo dos sobreviventes a idade variou de 28 a $78 \mathrm{com}$ média de 59,15 anos. Já em relação ao IMC no grupo dos não sobreviventes variou de 22 a 29 com média de 26 .
Enquanto no grupo dos sobreviventes a média foi de 25,6. Não houve diferença significativa entre idade e IMC no grupo dos sobreviventes em relação ao grupo dos não sobreviventes (Tabela 1).

Tabela 1 - Pacientes dos Grupos Sobrevivente e Não Sobrevivente conforme a Idade e o IMC

\begin{tabular}{cccc}
\hline \multicolumn{2}{c}{ Sobreviventes } & \multicolumn{2}{c}{ Não Sobreviventes } \\
\hline Idade & IMC & Idade & IMC \\
\hline 54 & 27,3 & 53 & 23,9 \\
67 & 25 & 43 & 25,1 \\
78 & 24 & 49 & 29,2 \\
69 & 22 & 51 & 24 \\
50 & 29,4 & 56 & 24 \\
52 & 24 & 44 & 24 \\
28 & 24 & 73 & 26 \\
51 & 27 & 39 & 22 \\
54 & 25 & 75 & 29 \\
33 & 23 & 81 & 24,2 \\
70 & 24 & 92 & 25 \\
70 & 24 & 92 & 31 \\
67 & 26 & 65 & 27 \\
45 & 25 & 85 & 31 \\
63 & 27 & 87 & 31 \\
68 & 27 & 59 & 23 \\
69 & 31 & 85 & 26 \\
77 & 29 & 70 & 23 \\
68 & 25 & & \\
50 & 25 & zcalc - 1,242 \\
\hline zcalc - 0,029 & & \\
\hline
\end{tabular}

Média de idade para os sobreviventes $=59,15$ e IMC de 26

Média de índice de massa corpórea (IMC) para os não sobreviventes $=66,61$ e IMC de 25,6

Teste de Mann-Whitney

No grupo dos não sobreviventes 15 foram submetidos a cirurgias de urgência, enquanto três foram eletivas. No grupo dos sobreviventes 14 pacientes foram submetidos a cirurgia eletiva, enquanto seis, urgência. Desta forma $55 \%$ das cirurgias foram de urgência.

O tempo de internação variou de 2 a 52 dias no grupo dos não sobreviventes com média de 11.3 dias. Enquanto no grupo dos sobreviventes a variação foi de 1 a 30 dias com média de 4,9. Em relação ao APACHE II no grupo dos não sobreviventes variou de 5 a $32 \mathrm{com}$ média de 19,14. Ao passo que no grupo dos sobreviventes o APACHE variou de 1 a 18 com média de 8,6. O risco de óbito no grupo dos não sobreviventes variou de 3,1 a 84,9 com média de 38,8; enquanto no grupo dos sobreviventes a média foi de 7,5. O tempo de internação, APACHE II e o risco de óbito apresentaram diferenças significativas em ambos os grupos, sendo maior no grupo dos não sobreviventes (Tabela 2). 
Tabela 2 - Pacientes Internados na UTI dos Grupos Sobrevivente e Não Sobreviventes conforme o Tempo de Internação. APACHE II e Risco de Óbito

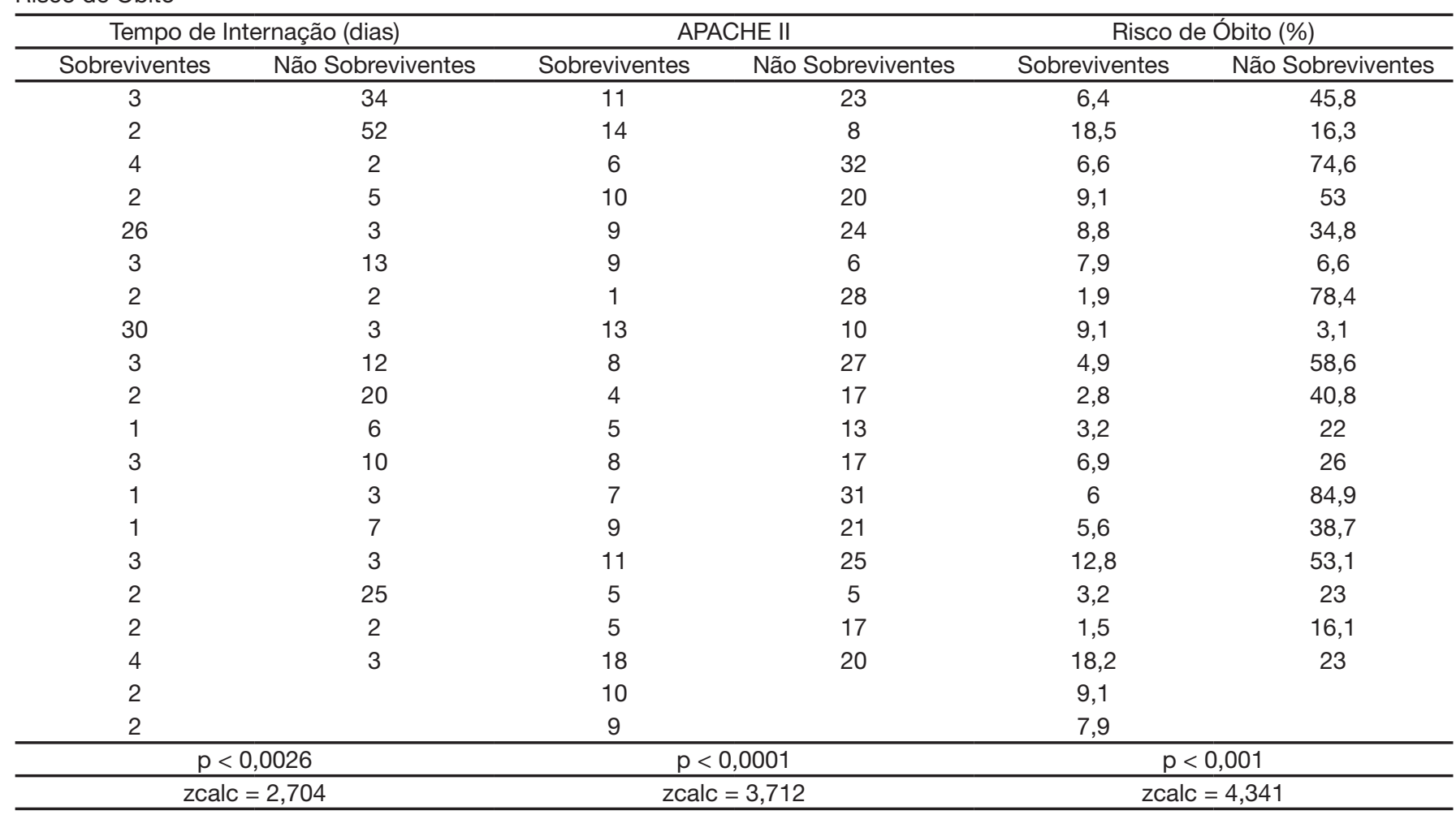

Tempo médio de internação - sobrevivente $=4,9$ e não sobrevivente $=11,3$ dias APACHE II médio - sobrevivente $=8,6$ e não sobrevivente $=19,14$

Risco de óbito médio - sobreviventes $=7,5$ e não sobreviventes $=38,82$

Teste de Mann-Whitney

A mortalidade geral foi de $47 \%$. A mortalidade dos pacientes com APACHE II maior do que 21 foi de $100 \%$, enquanto nos pacientes com APACHE entre 10 e 20 foi de $50 \%$. Nos pacientes com APACHE menor do que 9 a mortalidade foi de $18 \%$. Foi calculada a Standardized Mortality Rate (SMR razão da mortalidade observada pela predita) que neste estudo foi de 1,22 , indicando que a mortalidade real encontrava-se $20 \%$ acima da mortalidade prevista. A mortalidade prevista para os pacientes com APACHE II acima de 21 foi de 58,5\%; para os pacientes com APACHE entre 10 e 20 foi de $19 \%$ e para os pacientes com APACHE II menor do que 9 foi de $7 \%$ (Tabela 3).

Tabela 3 - Resumo das Tabelas Comparando as Médias das Variáveis Estudadas no Grupo dos Sobreviventes e Não Sobreviventes

\begin{tabular}{lccc}
\hline & Sobreviventes Não Sobreviventes & $\mathrm{p}$ \\
\hline $\begin{array}{l}\text { Idade (anos) } \\
\text { Índice de massa }\end{array}$ & 59,15 & 66,61 & \\
$\begin{array}{l}\text { corpórea } \\
\text { Tempo de inter- }\end{array}$ & 25,68 & 26,02 & \\
nação (dias) & 4,9 & 11,38 & $\mathrm{p}<0,0026$ \\
APACHE II & 8,6 & 19,14 & $\mathrm{p}<0,0001$ \\
Risco de óbito (\%) & 7,5 & 38,82 & $\mathrm{p}<0,001$ \\
\hline
\end{tabular}

\section{DISCUSSÃO}

Em estudo realizado para avaliar a aplicação do APACHE II em pacientes internados em UTI do Brasil, foram coletados dados prospectivos de 1781 pacientes admitidos em 10 UTI no período de janeiro de 1990 a maio de 1991. A razão da mortalidade observada pelo padrão foi de 1,66. Os resultados indicaram que o APACHE II foi capaz de estratificar pacientes conforme o risco de óbito ${ }^{8}$. Da mesma forma observou-se maior mortalidade no presente estudo, conforme o aumento do APACHE, com SMR de 1,22.

Realizou-se um estudo multicêntrico para avaliar a relação entre tempo de permanência na UTI e sobrevivência. Foram coletados dados de 5 hospitais num total de 8157 pacientes. A mortalidade dos pacientes com tempo de internação até 30 dias foi de $7 \%$ enquanto de pacientes com mais de 30 dias foi de $22 \%$. Com o aumento do tempo de internação, a sobrevivência nas UTI diminuiu, mas não chegou a zero. Concluiu-se que o tempo de internação prolongado não resulta rotineiramente falta de sobrevivência na UTI em estudo ${ }^{9}$. 
Neste estudo observou-se maior mortalidade nos pacientes que tiveram maior tempo de internação.

Estudo prospectivo realizado na UTI do Hospital Regional de São José Dr. Homero de Miranda Gomes, no período de janeiro a julho de 1996, com o objetivo de observar a aplicabilidade do APACHE II em pacientes cirúrgicos dessa unidade, foram avaliados 84 pacientes cirúrgicos que necessitaram de cuidados intensivos por período superior a 24 horas. A faixa etária ficou entre 56 e 65 anos. Quanto ao sexo, 61 pacientes eram do sexo masculino. Quanto às procedências, 56 pacientes foram submetidos à cirurgia de urgência $(66,67 \%)$ e $28(33,33 \%)$ à cirurgia eletiva. A taxa de mortalidade foi de $38,09 \%$ (32 pacientes). Em relação ao APACHE II, observou-se que o escore médio da amostra foi de $17,06 \pm 9,37$; no grupo dos sobreviventes o escore médio foi de 12,31 , enquanto o dos não sobreviventes foi de 24,78 ( $p<0,0003$ ). O risco de óbito da amostra total alcançou uma média de $24,57 \pm 28,80$; no grupo dos sobreviventes ficou em 11,47 e no grupo dos não sobreviventes 47,03 ( $p<0,00001$ ); SMR foi de 1,55. O sistema APACHE II mostrou-se um índice preditivo adequado de mortalidade em pacientes cirúrgicos que necessitaram de cuidados intensivos ${ }^{6}$. Neste estudo a média do APACHE dos não sobreviventes e sobreviventes foi maior, assim como a SMR.

Outro estudo avaliou o uso do APACHE II como um índice de previsão confiável da evolução de pacientes críticos em Singapura. Foram admitidos 130 pacientes no período de junho a novembro de 1991. A idade média foi de 53 , 4 com $51 \%$ homens. A média do tempo de internação foi de 4 dias e $62 \%$ dos pacientes necessitaram de ventilação mecânica. A média do APACHE II foi de 21,5. O APACHE II para os sobreviventes foi menor que o dos não sobreviventes. A mortalidade real foi de $37,4 \%$. Comparando pacientes abaixo e acima de 60 anos não houve diferença significativa na média do tempo de internação e APACHE $\|^{22}$. Da mesma forma o APACHE dos sobreviventes foi menor em relação aos não sobreviventes.

Realizou-se um estudo em Singapura onde foram avaliados 301 pacientes admitidos na unidade de terapia intensiva cirúrgica de julho de 1991 a maio de 1992. Foram avaliados 174 homens $(57,8 \%)$ e 127 mulheres $(42,2 \%)$. A idade média dos pacientes foi de 52,39 anos (variando de 13 a 93) e o tempo de internação médio foi de 5,37 dias (variando de 1 a 85). Cirurgia de urgência foi realizada em 91 pacientes (30,23\%). O índice APACHE II para os sobreviventes foi de 12,94 e não sobreviventes de $28,19^{10}$. Mais uma vez se observou relação do APA-
CHE maior para pacientes não sobreviventes.

Estudo realizado em hospital universitário na Alemanha, avaliou influência do sexo na evolução dos pacientes cirúrgicos na UTI. Foram coletados dados de 4218 pacientes críticos (2709 do sexo masculino e 1509 do sexo feminino). A indicação de cuidados intensivos foi menor no sexo feminino, levando a uma proporção estatisticamente menor de mulheres na UTI. Não houve diferença em relação à mortalidade por choque séptico entre os sexos ${ }^{11}$. Neste estudo o número de mulheres foi menor em relação ao de homens não podendo ser realizado nenhuma análise estatística para avaliar a relação do sexo e mortalidade .

Em estudo prospectivo, realizado durante 13 meses na UTI do Hospital São Paulo, foram selecionados 520 pacientes, nos quais foi aplicado o índice de prognóstico APACHE II. Foi observado que pacientes com APACHE II acima de 25 apresentaram evolução significativa para o óbito. O SMR foi de 1,20. Concluiu-se que o APACHE II é um índice que possibilita classificar pacientes críticos de acordo com a gravidade de sua condição ${ }^{1}$. Como em outros estudos o APACHE II maior tem correlação direta com aumento da mortalidade e o SMR foi semelhante ao observado ao observado neste estudo.

Estudo prospectivo realizado entre abril e julho de 2004, avaliando 185 pacientes submetidos a procedimentos programados ou de emergência admitidos em UTI cirúrgica, foram registrados idade, sexo altura e peso, temperatura, estado físico (ASA), tipo de intervenção cirúrgica, tempo de internação dentre outros. O tempo de internação foi de 4 a 10 dias. Os fatores de riscos significativos foram o tempo de internação na UTI e intervenções de emergência de grande porte. Assim observaram que pacientes com internações prolongadas em UTI estão associados às maiores mortalidades hospitalares ${ }^{12}$. Da mesma maneira, neste estudo a média do tempo de internação foi significativamente maior nos pacientes não sobreviventes.

Estudo avaliou o impacto do IMC na evolução dos pacientes críticos. Foram coletados, retrospectivamente, dados de 63646 pacientes, onde foi observou-se mortalidade aumentada em pacientes abaixo do peso (IMC $<20)^{13}$. Nesse estudo não houve diferença estatística entre o grupo dos sobreviventes e não sobreviventes em relação ao IMC.

Estudo prospectivo conduzido por dois anos em seis UTI na França, avaliou a associação entre mortalidade e IMC em paciente internados em UTI. Foram incluídos 1698 pacientes, aproximadamente a metade dos 
pacientes apresentaram IMC normal, 10\% estavam abaixo do peso e $40 \%$ com sobrepeso. Observou-se mortalidade significativamente maior em pacientes com IMC $<18,5^{14}$. Este trabalho entra em concordância com o anterior com aumento da mortalidade em relação ao IMC.

Em estudo realizado com 184 pacientes, para avaliar o efeito do IMC na evolução dos pacientes no Sentara Norfolk General Hospital, o IMC médio foi de 25,15. A mortalidade dos pacientes com IMC de 27 foi de 5\%, de $27 \%$ a $31,8 \%$. Pacientes com IMC maior que 31 foi de $42,1 \%$, isso foi avaliado como sendo associado à maior número de complicações pulmonares em relação aos outros grupos ${ }^{15}$. Este trabalho numa população diferente apresentou resultados contraditórios em relação aos anteriores. Na casuística do Hospital Grajaú não houve diferença significativa em relação ao IMC no grupo dos sobreviventes e não sobreviventes. Em pacientes graves qualquer parâmetro que inclui peso não é confiável, pois apesar da extensa perda de massa magra, eles apresentam um aumento na composição, por aumento da água corpórea ${ }^{16}$. São considerados parâmetros de avaliação nutricional: dobra cutânea do tríceps, circunferência do braço e circunferência muscular do braço ${ }^{17}$, transferrina ${ }^{18}$, albumina ${ }^{19}$, colesterol sérico ${ }^{20}$, linfocitometria ${ }^{21}$, balanço nitrogenado ${ }^{19}$ e índice creatinina altura ${ }^{20}$. A proteína-C reativa e a alfa -glicoproteína $_{1}$ ácida são proteínas da fase aguda do estresse metabólico que têm relação com o prognóstico nutricional ${ }^{18}$.

\section{CONCLUSÃO}

Diante do exposto, os pacientes não sobreviventes apresentaram APACHE II significativamente maior que os sobreviventes, demonstrando ser um índice prognóstico eficaz condizente com a literatura. A SMR encontrou-se dentro da média observada na literatura. Houve maior tempo de internação dos pacientes não sobreviventes em relação aos sobreviventes.

Não houve diferença estatística em relação ao IMC nos dois grupos, necessitando-se de novos estudos para avaliar a relação do estado nutricional e mortalidade.

\section{REFERÊNCIAS}

01. Costa JI, Gomes do Amaral JL, Munechika M et al - Severity and prognosis in intensive care: prospective application of the APACHE II index. São Paulo Med J, 1999;117:205-214.

02. Schettino G, Cardoso LF, Junior JM et al - Pacientes Críticos Diagnóstico e Tratamento Hospital Sírio-Libanês. $1^{\text {a }}$ Ed, Barueri, Editora Manole, 2006.

03. Ponce de Leon-Rosales SP, Molinar-Ramos F, Dominguez-Cherit G et al - Prevalence of infections in intensive care units in Mexico: a multicenter study. Crit Care Med, 2000;28:1316:1321.

04. Knaus WA, Draper EA, Wagner DP et al. APACHE II: A severity of disease classification system . Crit Care Med ,1985; 13: 818-29

05. Kruse JA, Thill-Baharozian MC, Carlson RW - Comparison of clinical assessment with APACHE II for predicting mortality risk in patients admitted to a medical intensive care unit. JAMA 1998;260:1739-1742.

06. Gonçalves WM, Kruel NF - Análise do sistema prognóstico de mortalidade APACHE II em pacientes cirúrgicos de unidade de terapia intensiva. Revista do Colégio Brasileiro de Cirurgiões, 1999;26:115-118

07. Siegel S, Castellan JR - Nonparametric Statistics. $2^{\text {nd }}$ Ed, New York, McGraw Hill Int Ed, 1988.

08. Bastos PG, Sun X, Knaus WA et al - Application of APACHE scoring system in brazilian ICUs. Crit Care Med, 1992;20:(4):S79

09. Fakhry S, Rutledge R, Rutherford E et al - No association of length of ICU stay with ICU survival: a 5 hospital multi-institutional study of 8157 surgical patients. Crit Care Med, 1994;22:A59.

10. Chen FG, Koh KF, Goh MH - Validation of APACHE II in surgical intensive care unit. Singapore Med J, 1993;34:322-324.

11. Wichmann MW, Inthorn D, Andress $\mathrm{HJ}$ et al - Incidence and mortality of severe sepsis in surgical intensive care patients: the influence of patient gender on disease process and outcome. Intensive Care Med, 2000;26:167-172.

12. Abelha FJ, Castro MA, Landeiro NM et al - Mortalidade e o tempo de internação em uma unidade de terapia intensiva cirúrgica. Rev Bras Anestesiol, 2006;56.34-45.

13. Tremblay A, Bandi V. Impact of body mass index on outcomes following critical care. Chest 2003;123:1202-1207.

14. Garrouste-Orgeas M, Troche G, Azoulay E et al - Body mass index. An additional prognostic factor in ICU patients. Intensive Care Med, 2004;30:437-443.

15. Choban PS, Weireter LJ Jr, Maynes C - Obesity and increased mortality in blunt trauma. J Trauma, 1991;31:1253-1257.

16. Ravasco P, Camilo ME, Gouveia-Oliveira A et al - A critical approach to nutritional assessment in critically ill patients. Clin Nutr, 2002;21:73-77.

17. Waizberg D et al. Inquérito Brasileiro de Avaliação Nutricional Hospitalar. São Paulo, 1996. (favor informar o nome de três autores e o nome da editora).

18. Waizberg DL - Nutrição Oral, Enteral e Parenteral na Prática Clínica. São Paulo, Atheneu, 2000.

19. Palma AGC - Avaliação Nutricional do Paciente Hospitalizado. Desafios e Limitações Clínicas. Revista Brasileira de Nutrição Clínica, 2005;20.

20. Bottoni A, Oliveira GPC, Ferrini MT et al - Avaliação Nutricional: Exames Laboratoriais. Nutrição Oral, Enteral e Parenteral na Prática Clínica. $3^{a}$ Ed, São Paulo: Atheneu, 2000.

21. Lee KH, Hui KP, Lim TK et al - Acute physiology and chronic health evaluation (APACHE II) scoring in the Medical Intensive Care Unit. Singapore Med J, 1993;34:41-44. 